\title{
Data report: updated magnetostratigraphy for IODP Sites U1403, U1408, U1409, and U1410'
}

\author{
Yuhji Yamamoto, ${ }^{2}$ Hiroto Fukami, ${ }^{2}$ Wakana Taniguchi, ${ }^{2}$ and Peter C. Lippert $^{3}$
}

\section{Chapter contents}

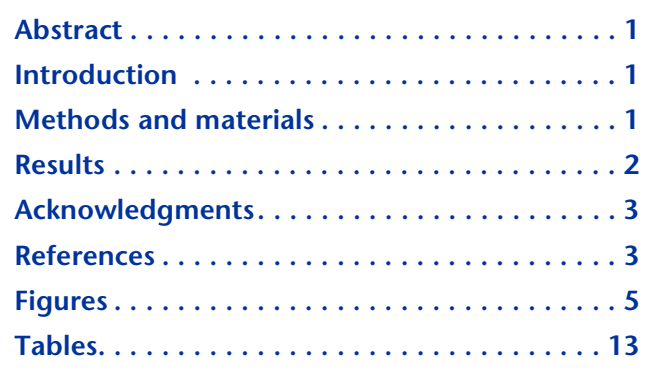

'Yamamoto, Y., Fukami, H., Taniguchi, W., and Lippert, P.C., 2018. Data report: updated magnetostratigraphy for IODP Sites U1403, U1408, U1409, and U1410. In Norris, R.D., Wilson, P.A., Blum, P., and the Expedition 342 Scientists, Proceedings of the Integrated Ocean Drilling Program, 342: College Station, TX (Integrated Ocean Drilling Program).

doi:10.2204/iodp.proc.342.207.2018

${ }^{2}$ Center for Advanced Marine Core Research, Kochi University, Kochi 783-8502, Japan. Correspondence author: y.yamamoto@kochi-u.ac.jp ${ }^{3}$ Department of Geology and Geophysics, University of Utah, Salt Lake City, UT 84112, USA.

\section{Abstract}

We conducted detailed paleomagnetic measurements on the sedimentary sections recovered at Integrated Ocean Drilling Program (IODP) Sites U1403, U1408, U1409, and U1410 during Expedition 342 to update the shipboard magnetostratigraphy. The intervals with negative inclinations are more clearly defined than the shipboard results, and this result has allowed us to refine chron boundary depths. In most cases, the chron boundary depths determined in the present study are not substantially different from the shipboard ones (less than $\sim 1 \mathrm{~m}$ ), but some boundaries have large differences. We also identify some new reversal boundaries that could not be resolved on board.

\section{Introduction}

Integrated Ocean Drilling Program (IODP) Expedition 342 recovered $\sim 5.4 \mathrm{~km}$ of hemipelagic sediment drifts from the J-Anomaly and Southeast Newfoundland Ridge in the northwest Atlantic, off the coast of Newfoundland. Routine shipboard measurements were used to develop magneto- and biostratigraphic age models (see the "Expedition 342 summary" chapter [Norris et al., 2014a]). The shipboard magnetostratigraphy was based on the measurement of natural remanent magnetization (NRM) of the archive-half cores after $20 \mathrm{mT}$ alternating field (AF) demagnetization on a wide-bore superconducting rock magnetometer in a magnetically noisy laboratory environment. In the present study, we performed detailed shore-based paleomagnetic measurements on the sedimentary sections recovered at Sites U1403, U1408, U1409, and U1410 (Fig. F1) to test and improve the shipboard magnetostratigraphy.

\section{Methods and materials}

U-channel samples (typically $1.5 \mathrm{~m}$ in length with a $2 \mathrm{~cm} \times 2 \mathrm{~cm}$ cross section) were taken from the central part of the archive-half cores along the stratigraphic splice described in the "Expedition 342 summary" chapter (Norris et al., 2014a): 30-160 m core composite depth below seafloor (CCSF) for Site U1403, 18-166 m CCSF for Site U1408, 6-142 m CCSF for Site U1409, and 0-165 m CCSF for Site U1410. A total of 530 samples were collected from archive halves, and 133 samples were collected from working 
halves. All samples were taken from intervals recovered using the advanced piston corer (APC) system. The samples were collected during February 2013 and March 2014 at the IODP Bremen Core Repository (Bremen, Germany). We conducted progressive AF demagnetizations on NRMs of the samples up to $80 \mathrm{mT}$ in approximately 10-12 treatment steps. The remanence was measured at each AF step at a stratigraphic resolution of $1 \mathrm{~cm}$. The $0-20 \mathrm{mT}$ demagnetization steps were omitted for archive-half samples because they were already demagnetized during the expedition.

Discrete samples of $7 \mathrm{~cm}^{3}$ cubes were collected during Expedition 342 every few meters along the working-half cores of Hole A at each site. These samples were also AF demagnetized up to $20 \mathrm{mT}$ to construct the shipboard magnetostratigraphy. To further constrain some of the chron boundaries in intervals recovered with the extended core barrel (XCB) system, the samples collected from 210 to $252 \mathrm{~m}$ CCSF (Site U1408) and 154 to $294 \mathrm{~m}$ CCSF (Site U1410) were subjected to additional progressive AF demagnetization from 20 to $80 \mathrm{mT}$ in $16-18$ steps.

All of the shore-based measurements were conducted using a cryogenic magnetometer with an in-line static AF demagnetizer (2G Enterprises model 760R) at the Center for Advanced Marine Core Research, Kochi University. This magnetometer has a response function (single peaks with their full widths at half maxima) of $46 \mathrm{~mm}(X$ and $Y$ ) and $54 \mathrm{~mm}(Z)$ (Oda et al., 2016), and measurement results are smoothed according to the function.

Characteristic remanent magnetization (ChRM) directions for discrete samples and at $1 \mathrm{~cm}$ intervals for the U-channel samples were calculated using principal component analysis (PCA; Kirschvink, 1980). No attempt was made to deconvolve the U-channel magnetic data (cf. Oda and Shibuya, 1996; Jackson et al., 2010; Oda and Xuan, 2014). Data from the top and bottom $5 \mathrm{~cm}$ of the U-channel samples and from disturbed intervals described in the expedition 342 site reports and observed in core photos were eliminated from the analysis (see the "Methods" chapter [Norris et al., 2014b]). We use the splice tables from the "Site U1403" chapter (Norris et al., 2014c) for Site U1403, by Hull et al. (2017) for Sites U1408 and U1409, and by Boulila et al. (2018) for Site U1410 to plot our data on a composite depth scale (CCSF).

\section{Results}

ChRM directions were typically resolved over five consecutive treatment steps after AF demagnetization of $20-40 \mathrm{mT}$. The origin of orthogonal vector plots was included as an additional data point in the PCA analysis, and the resulting maximum angle of deviation (MAD) was $<10^{\circ}$ for most discrete samples and most intervals of the U-channel samples. Representative orthogonal vector plots of the AF demagnetization results are shown in Figures F2 (U-channel) and F3 (discrete). The resultant ChRM directions and associated parameters are shown in Tables T1 and T2 for the discrete samples. Although few samples or intervals were completely demagnetized by $80 \mathrm{mT}$, the straight-line decay toward the origin of the orthogonal vector plots gives us confidence that the data accurately distinguish the ChRM.

The ChRM directions resulted in absolute inclinations and relative declinations. The relative declination is provided as an angular departure of the ChRM from the double line of a core liner in the $X-Y$ plane of the sample coordinate (see Fig. F6 in the "Methods" chapter [Norris et al., 2014b]). We observed that changes in the inclinations between negative and positive values are usually associated with $\sim 180^{\circ}$ azimuthal changes in the relative declinations for each APC core. This relationship implies that intervals with opposite declinations record opposite geomagnetic polarity. If we assume that the relative declinations mainly correspond to geographic north (south) when the inclinations are positive (negative), then we can convert them into "corrected" declinations by rotating the relative declinations for each core such that the mean declination of the normal and inverted reversed polarity intervals is zero.

The FlexIt orientation tool was deployed during the recovery of some APC cores. The magnetic tool face (MTF) orientation value gives the angle between geomagnetic north and the double line on the core liner for each core (see the "Methods" chapter [Norris et al., 2014b]). The sum of the MTF angle and the mean declination for each core equals $7.9^{\circ} \pm 38.3^{\circ}(1 \sigma, N=$ 87; Table T3; Fig. F4). On average, the summed angle is indistinguishable from the present declination of approximately $-17^{\circ}$ at the drill sites according to the twelfth generation of the International Geomagnetic Reference Field model (Thébault et al., 2015). We used the inclinations and the corrected declinations to calculate virtual geomagnetic pole (VGP) latitudes at a resolution of $1 \mathrm{~cm}$ along each APC core section. Note that because of the response function of the magnetometer, the data are inherently smoothed over a $10 \mathrm{~cm}$ stratigraphic window. Downhole variations of these results are shown in Figures F5, F6, F7, and F8 for the four sites.

These figures show that APC intervals with negative inclinations are more clearly defined in shore-based results than they are in the shipboard results. We attribute this to several factors, including more thor- 
ough removal of a viscous drilling overprint, a magnetically quieter measurement environment, and a higher sensitivity magnetometer. These better-resolved negative inclinations allow us to locate chron boundary depths more precisely and with greater confidence. We used a threshold VGP latitude of $40^{\circ}$ to define intervals of distinct polarity and determined the boundary depths based on the downhole variations of VGP latitude.

For intervals 210-252 $\mathrm{m} \mathrm{CCSF}$ at Site U1408 and 154-294 m CCSF at Site U1410 (XCB intervals), the downhole variations of inclination are somewhat different between the results obtained from the discrete samples and the shipboard results (Figs. F6, F8). This difference is probably because the shipboard results are heavy disturbed during the drilling by $\mathrm{XCB}$ (such as biscuits). We determined the chron boundary depths based on switching in signs of inclinations derived from the discrete samples.

We updated the shipboard magnetostratigraphy (see the "Expedition 342 summary" chapter [Norris et al., 2014a]) for each of the four sites studied here. For our correlations we relied on the 2012 geomagnetic polarity timescale (GPTS; Ogg, 2014) and on biostratigraphic datums reported in the "Expedition 342 summary" chapter (Norris et al., 2014a). The magnetostratigraphy for each site is shown in the rightmost panel in Figures F5, F6, F7, and F8 and is summarized in Tables T4, T5, T6, and T7.

In most cases, the chron boundary depths determined in the present study are very similar to those determined during Expedition 342 (differences less than $\sim 1 \mathrm{~m}$ ). However, there are some boundaries with large differences: $+3.79 \mathrm{~m}$ for $\mathrm{C} 18 \mathrm{r} / \mathrm{C} 19 \mathrm{n}$ in Hole U1403B (Table T4), $-2.14 \mathrm{~m}$ for C18n.2n/C18r in Hole U1408C (Table T5), +3.73 m for C19r/C20n in Hole U1408B (Table T5), and $+4.77 \mathrm{~m}$ for C2Or/ C21n in Hole U1409C (Table T6).

In the present study, we also identified several chron boundaries that were not identified during Expedition 342: C18n.2n/C18r in Hole U1408B (Table T5); C17n.2r/C17n.3n, C17n.3n/C17r, and C17r/C18n.1n in Hole U1408C (Table T5); C18r/C19n and C19n/ C19r in Hole U1409B (Table T6); C2n/C2r.1r and C20n/C20r in Hole U1410B (Table T7); and C18n.1r/ C18n.2n in Hole U1410C (Table T7).

\section{Acknowledgments}

This research used samples and data provided by the Integrated Ocean Drilling Program (IODP). The authors are indebted to the staff of the R/V JOIDES Resolution and the Bremen Core Repository and also gratefully acknowledge the Expedition 342 science party. We thank Katerina Petronotis for careful reviews to improve the report. Funding for this research was provided by the Japan Agency for MarineEarth Science and Technology (JAMSTEC) IODP Expedition 342 After Cruise Research Program, the Japan Society for the Promotion of Science (JSPS) KAKENHI (16H04043), and the Kochi University Research Project (Earth Investigation Project: Past, present, and future of environment, earthquake, and resources recorded in the ocean and land).

\section{References}

Boulila, S., Vahlenkamp, M., De Vleeschouwer, D., Laskar, J., Yamamoto, Y., Pälike, H., Tuner, S.K., Sexton, P.F., Westerhold, T., and Röhl, U., 2018. Towards a robust and consistent middle Eocene astronomical timescale. Earth and Planetary Science Letters, 486:94-107. https://doi.org/10.1016/j.epsl.2018.01.003

Hull, P.M., Bohaty, S.M., Cameron, A., Coxall, H.K., D'haenens, S., De Vleeschouwer, D., Elder, L.E., Friedrich, O., Kerr, K., Turner, S.K., Kordesch, W.E.C., Moriya, K., Norris, R.D., Opdyke, B.N., Penman, D.E., Pälike, H., Wilson, P.A., Sexton, P.F., Vahlenkamp, M., Wu, F., and Zachos, J.C., 2017. Data report: coarse fraction record for the Eocene megasplice at IODP Sites U1406, U1408, U1409, and U1411. In Norris, R.D., Wilson, P.A., Blum, P., and the Expedition 342 Scientists, Proceedings of the Integrated Ocean Drilling Program, 342: College Station, TX (Integrated Ocean Drilling Program). https://doi.org/10.2204/iodp.proc.342.203.2017

Jackson, M., Bowles, J.A., Lascu, I., and Solheid, P., 2010. Deconvolution of U-channel magnetometer data: experimental study of accuracy, resolution, and stability of different inversion methods. Geochemistry, Geophysics, Geosystems, 11(7):1-21.

https://doi.org/10.1029/2009gc002991

Kirschvink, J.L., 1980. The least-squares line and plane and the analysis of palaeomagnetic data. Geophysical Journal of the Royal Astronomical Society, 62(3):699-718. http:// dx.doi.org/10.1111/j.1365-246X.1980.tb02601.x

Norris, R.D., Wilson, P.A., Blum, P., Fehr, A., Agnini, C., Bornemann, A., Boulila, S., Bown, P.R., Cournede, C., Friedrich, O., Ghosh, A.K., Hollis, C.J., Hull, P.M., Jo, K., Junium, C.K., Kaneko, M., Liebrand, D., Lippert, P.C., Liu, Z., Matsui, H., Moriya, K., Nishi, H., Opdyke, B.N., Penman, D., Romans, B., Scher, H.D., Sexton, P., Takagi, H., Turner, S.K., Whiteside, J.H., Yamaguchi, T., and Yamamoto, Y., 2014a. Expedition 342 summary. In Norris, R.D., Wilson, P.A., Blum, P., and the Expedition 342 Scientists, Proceedings of the Integrated Ocean Drilling Program, 342: College Station, TX (Integrated Ocean Drilling Program).

http://dx.doi.org/10.2204/iodp.proc.342.101.2014

Norris, R.D., Wilson, P.A., Blum, P., Fehr, A., Agnini, C., Bornemann, A., Boulila, S., Bown, P.R., Cournede, C., Friedrich, O., Ghosh, A.K., Hollis, C.J., Hull, P.M., Jo, K., 
Junium, C.K., Kaneko, M., Liebrand, D., Lippert, P.C., Liu, Z., Matsui, H., Moriya, K., Nishi, H., Opdyke, B.N., Penman, D., Romans, B., Scher, H.D., Sexton, P., Takagi, H., Turner, S.K., Whiteside, J.H., Yamaguchi, T., and Yamamoto, Y., 2014b. Methods. In Norris, R.D., Wilson, P.A., Blum, P., and the Expedition 342 Scientists, Proceedings of the Integrated Ocean Drilling Program, 342: College Station, TX (Integrated Ocean Drilling Program). http://dx.doi.org/10.2204/iodp.proc.342.102.2014

Norris, R.D., Wilson, P.A., Blum, P., Fehr, A., Agnini, C., Bornemann, A., Boulila, S., Bown, P.R., Cournede, C., Friedrich, O., Ghosh, A.K., Hollis, C.J., Hull, P.M., Jo, K., Junium, C.K., Kaneko, M., Liebrand, D., Lippert, P.C., Liu, Z., Matsui, H., Moriya, K., Nishi, H., Opdyke, B.N., Penman, D., Romans, B., Scher, H.D., Sexton, P., Takagi, H., Turner, S.K., Whiteside, J.H., Yamaguchi, T., and Yamamoto, Y., 2014c. Site U1403. In Norris, R.D., Wilson, P.A., Blum, P., and the Expedition 342 Scientists, Proceedings of the Integrated Ocean Drilling Program, 342: College Station, TX (Integrated Ocean Drilling Program).

http://dx.doi.org/10.2204/iodp.proc.342.104.2014

Oda, H., and Shibuya, H., 1996. Deconvolution of longcore paleomagnetic data of Ocean Drilling Program by Akaike's Bayesian Information Criterion minimization. Journal of Geophysical Research: Solid Earth, 101(B2):2815-2834.

http://dx.doi.org/10.1029/95JB02811
Oda, H., and Xuan, C., 2014. Deconvolution of continuous paleomagnetic data from pass-through magnetometer: a new algorithm to restore geomagnetic and environmental information based on realistic optimization. Geochemistry, Geophysics, Geosystems, 15(10):39073924. https://doi.org/10.1002/2014GC005513

Oda, H., Xuan, C., and Yamamoto, Y., 2016. Toward robust deconvolution of pass-through paleomagnetic measurements: new tool to estimate magnetometer sensor response and laser interferometry of sample positioning accuracy. Earth, Planets and Space, 68:109. https://doi.org/10.1186/s40623-016-0493-2

Ogg, J.G., 2012. Geomagnetic polarity time scale. In Gradstein, F.M., Ogg, J.G., Schmitz, M.D., and Ogg, G.M. (Eds.), The Geologic Time Scale 2012: Amsterdam (Elsevier), 85-113. http://dx.doi.org/10.1016/B978-0-44459425-9.00005-6

Thébault, E., Finlay, C.C., Beggan, C.D., Alken, P., Aubert, J., Barrois, O., Bertrand, F., et al., 2015. International Geomagnetic Reference Field: the 12th generation. Earth, Planets and Space, 67:79.

https://doi.org/10.1186/s40623-015-0228-9

Initial receipt: 17 June 2017

Acceptance: 4 April 2018

Publication: 25 May 2018

MS 342-207 
Figure F1. Map showing Expedition 342 drill Sites U1403, U1408, U1409, and U1410.

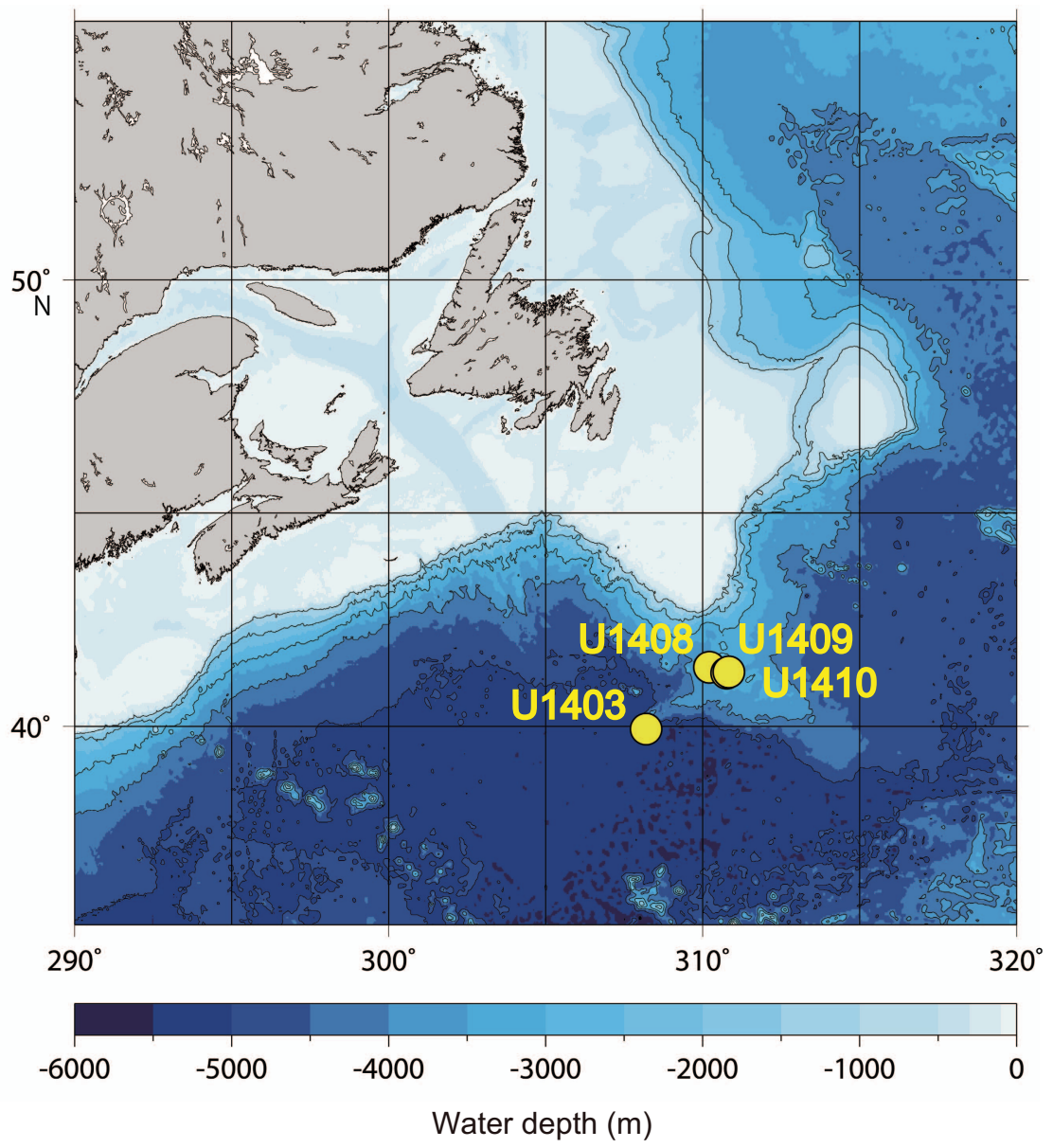


Figure F2. Representative orthogonal vector plots of AF demagnetization results from the U-channel samples. ChRM directions for (A, B) Site U1403, (C, D) Site U1408, (E, F) Site U1409, and (G, H) Site U1410. Solid circles $=$ horizontal projections, open circles $=$ vertical projections, shaded circles $=$ data not used in PCA analyses, black dashed lines $=$ best fit to the data. Inc $=$ inclination $\left({ }^{\circ}\right)$, rel. $\mathrm{Dec}=$ relative declination $\left({ }^{\circ}\right), \operatorname{MAD}=$ maximum angle of deviation $\left({ }^{\circ}\right)$.

A U1403A-12H-4A, $115 \mathrm{~cm}$

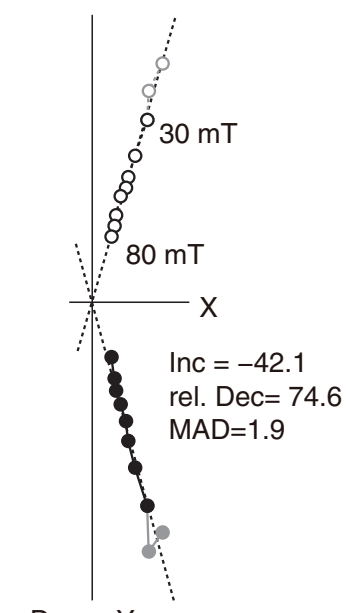

Down, $Y$

E

U1409A-8H-4A, $14 \mathrm{~cm}$

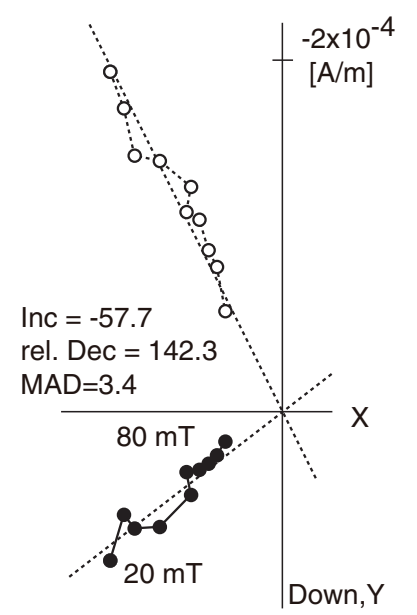

B

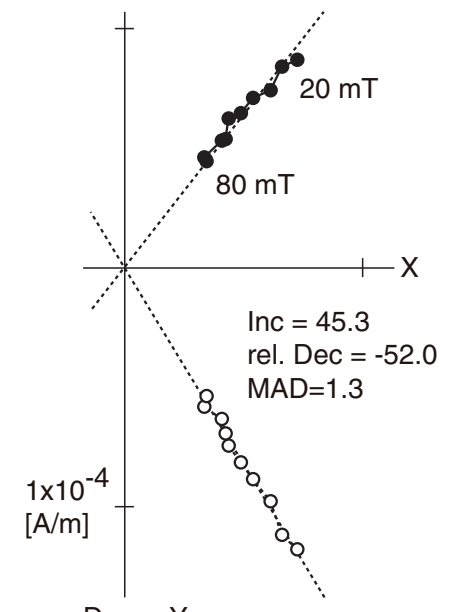

$\mathbf{F}$

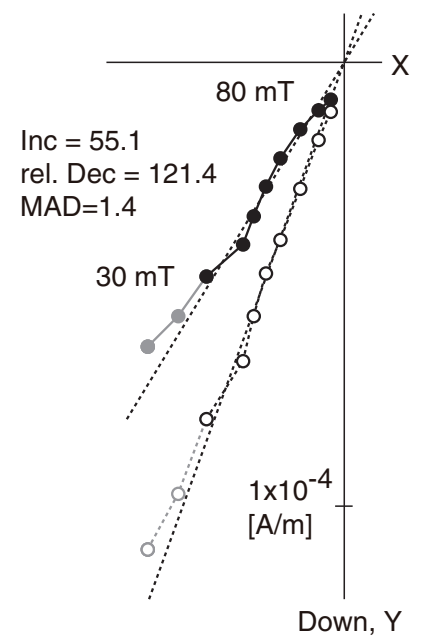

C U1408A-14H-4A, $40 \mathrm{~cm}$

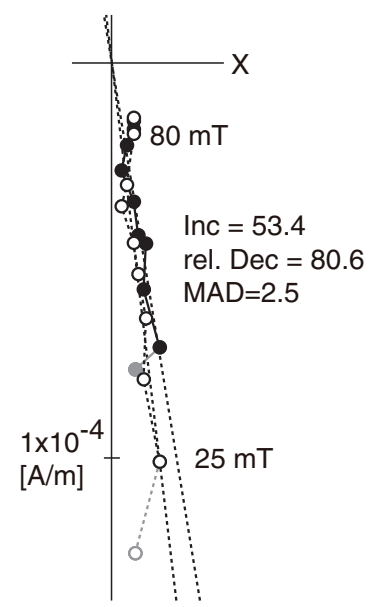

Down, Y

G

U1410A-11H-3A, $37 \mathrm{~cm}$

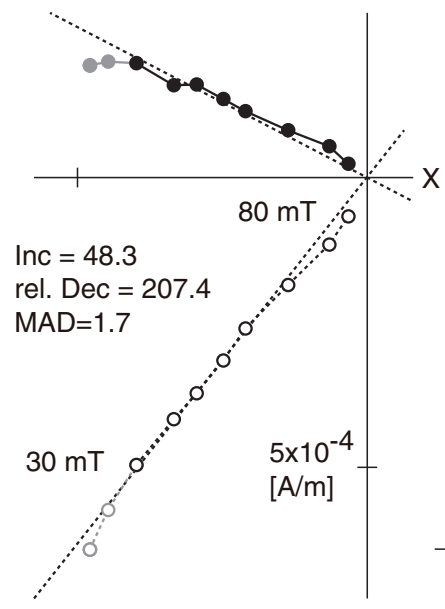

Down, Y
D U1408B-13H-4W, $50 \mathrm{~cm}$

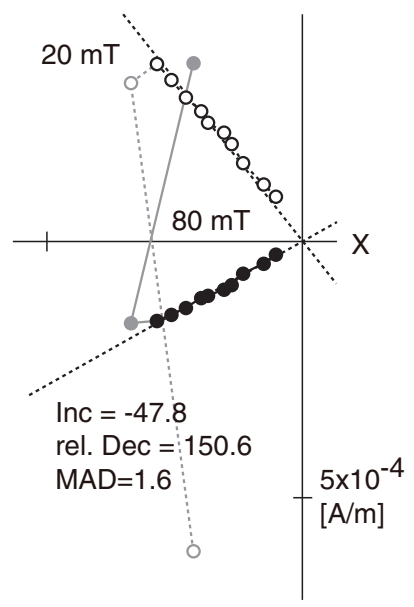

Down, $Y$

H

1410B-14H-5A, $104 \mathrm{~cm}$

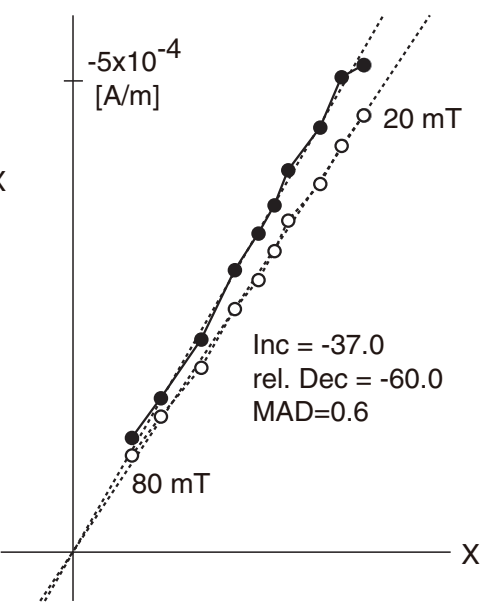

Down, Y 
Figure F3. Representative orthogonal vector plots and NRM decay plots of the AF demagnetization results from the discrete samples. ChRM directions for (A, B) Site U1408 and (C, D) Site U1410. Solid circles = horizontal projections, open circles $=$ vertical projections, gray circles $=$ data not used in PCA analyses, black dashed lines $=$ best-fit to the data. Inc $=$ inclination $\left({ }^{\circ}\right)$, rel. Dec $=$ relative declination $\left({ }^{\circ}\right), \mathrm{MAD}=$ maximum angle of deviation $\left({ }^{\circ}\right)$.

A

U1408A-21X-6W, 78-80 cm

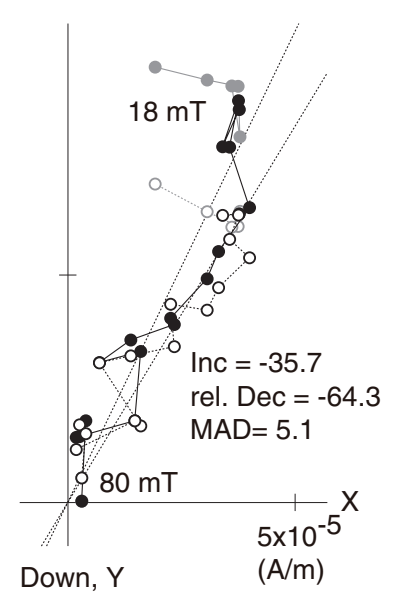

C

U1410A-22X-3W, 70-72 cm

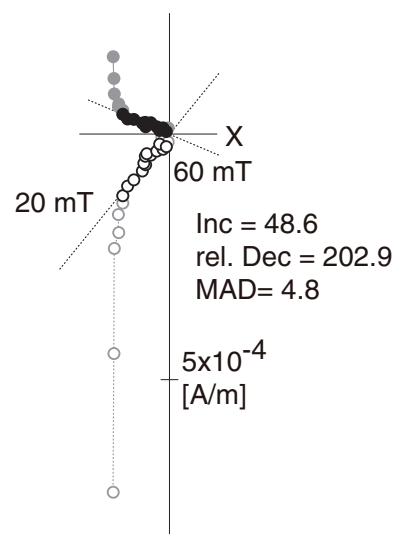

Down, $Y$
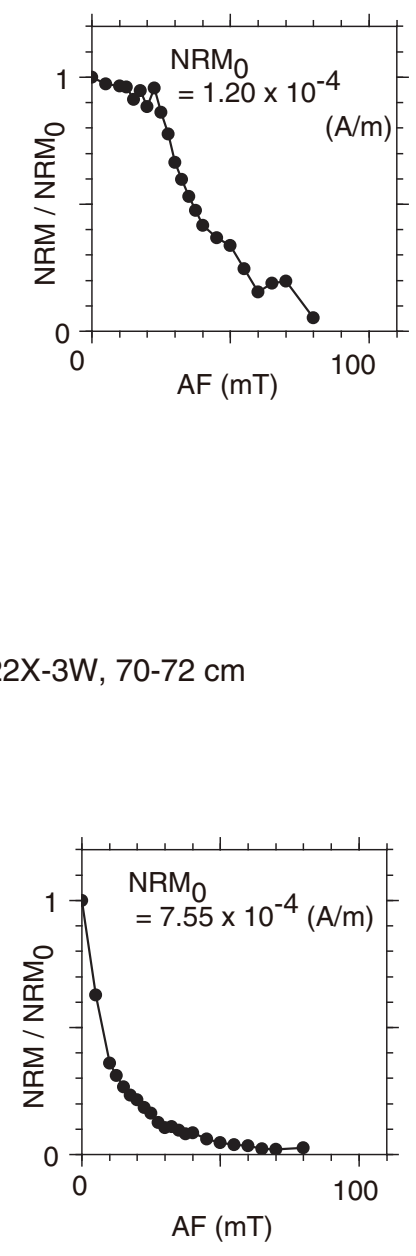

Proc. IODP | Volume 342
B

U1408A-25X-1W, 85-87 cm
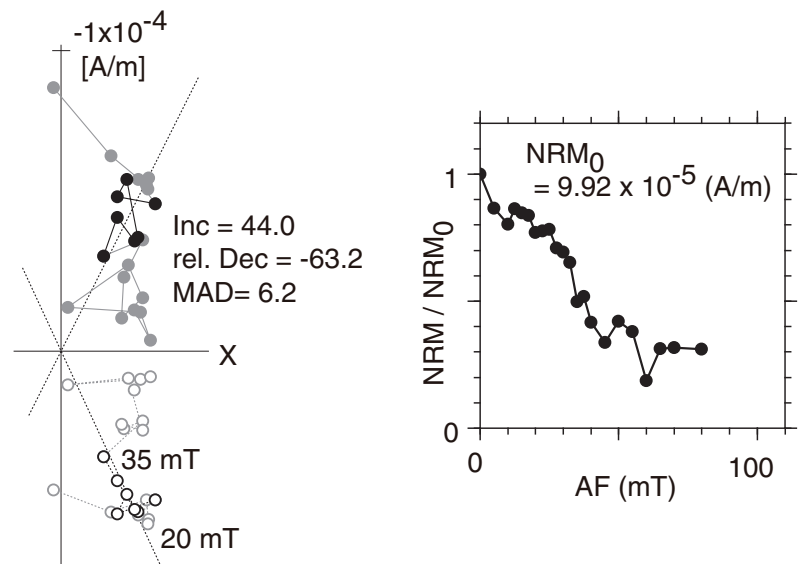

Down, Y
D

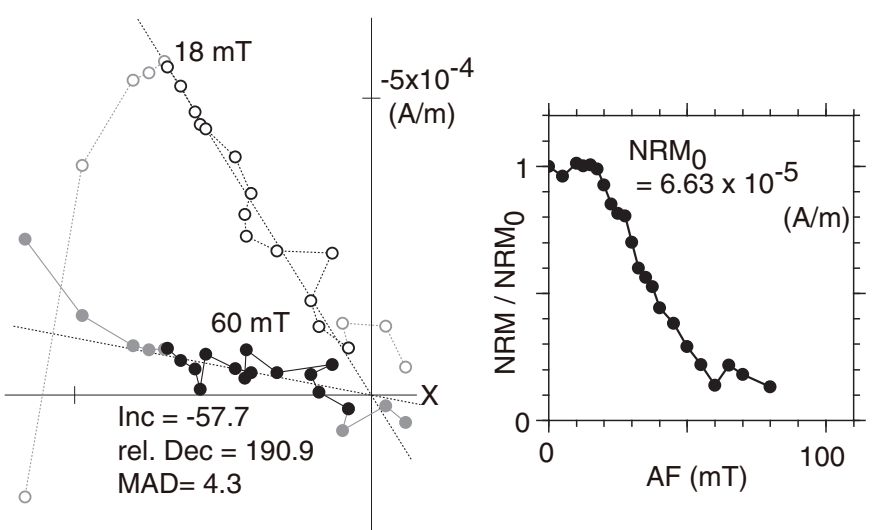

Down, Y
U1410A-24X-5W, 60-62 cm 
Figure F4. Histogram for the summed angle of the magnetic tool face (MTF) and the mean declination for each core. Eighty-seven data values resulted in an average of $7.9^{\circ}$ and a standard deviation of $38.3^{\circ}$, indicating that the mean declination is indistinguishable from today's declination of approximately $-17^{\circ}$ at the core recovery sites. ChRM = characteristic remanent magnetization.

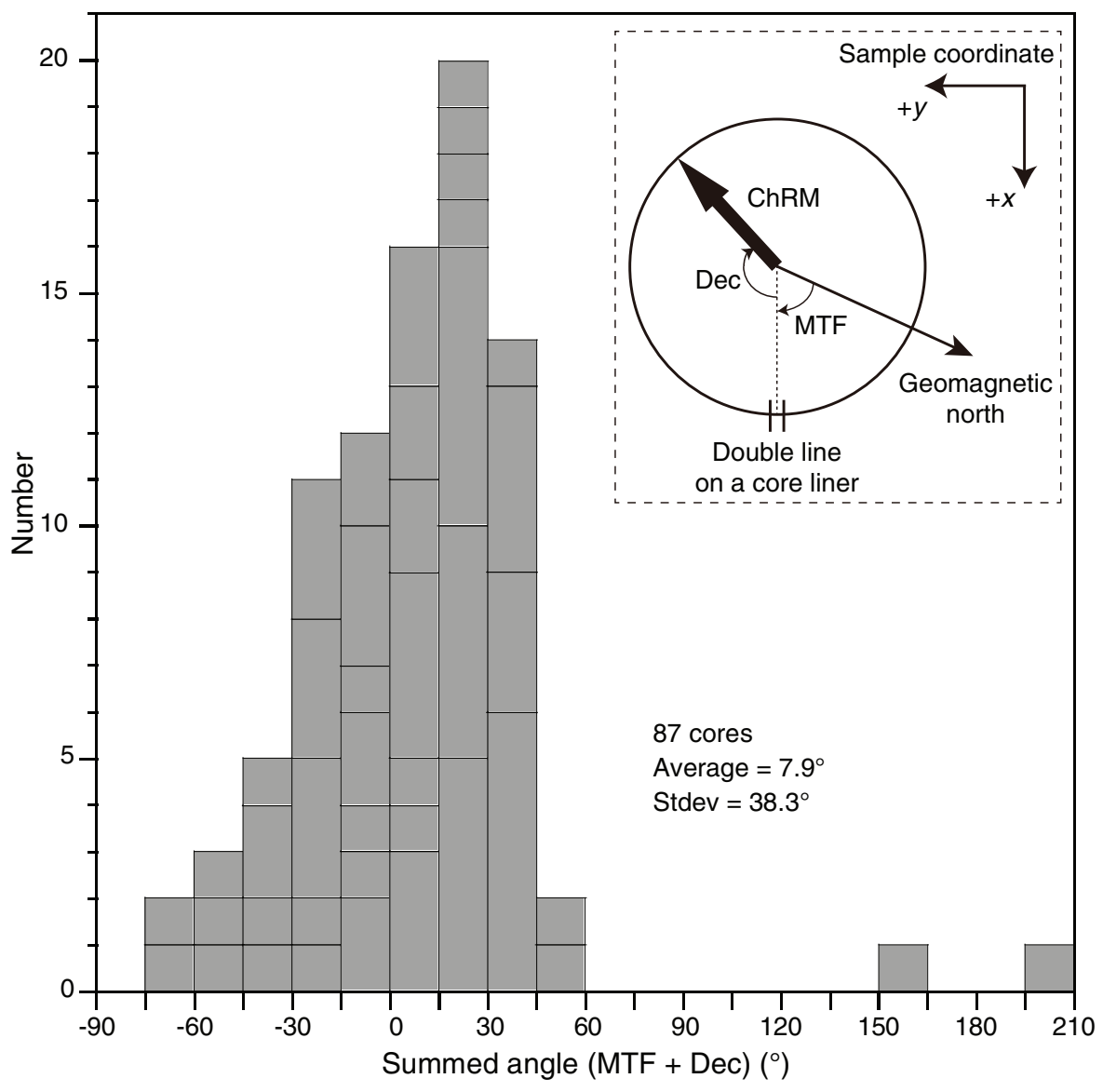


Figure F5. Plots of downhole variation of NRM intensity after $20 \mathrm{mT}$ demagnetization, PCA inclination, corrected PCA declination, maximum angular deviation (MAD) and virtual geomagnetic pole (VGP) latitude for Site U1403. Pink $=$ Hole U1403A; green $=$ Hole U1403B. Shaded $=$ shipboard data Interpreted paleomagnetic polarities and chrons are indicated in the rightmost panel (black $=$ normal, white $=$ reversed, shaded $=$ unidentified).

Site U1403

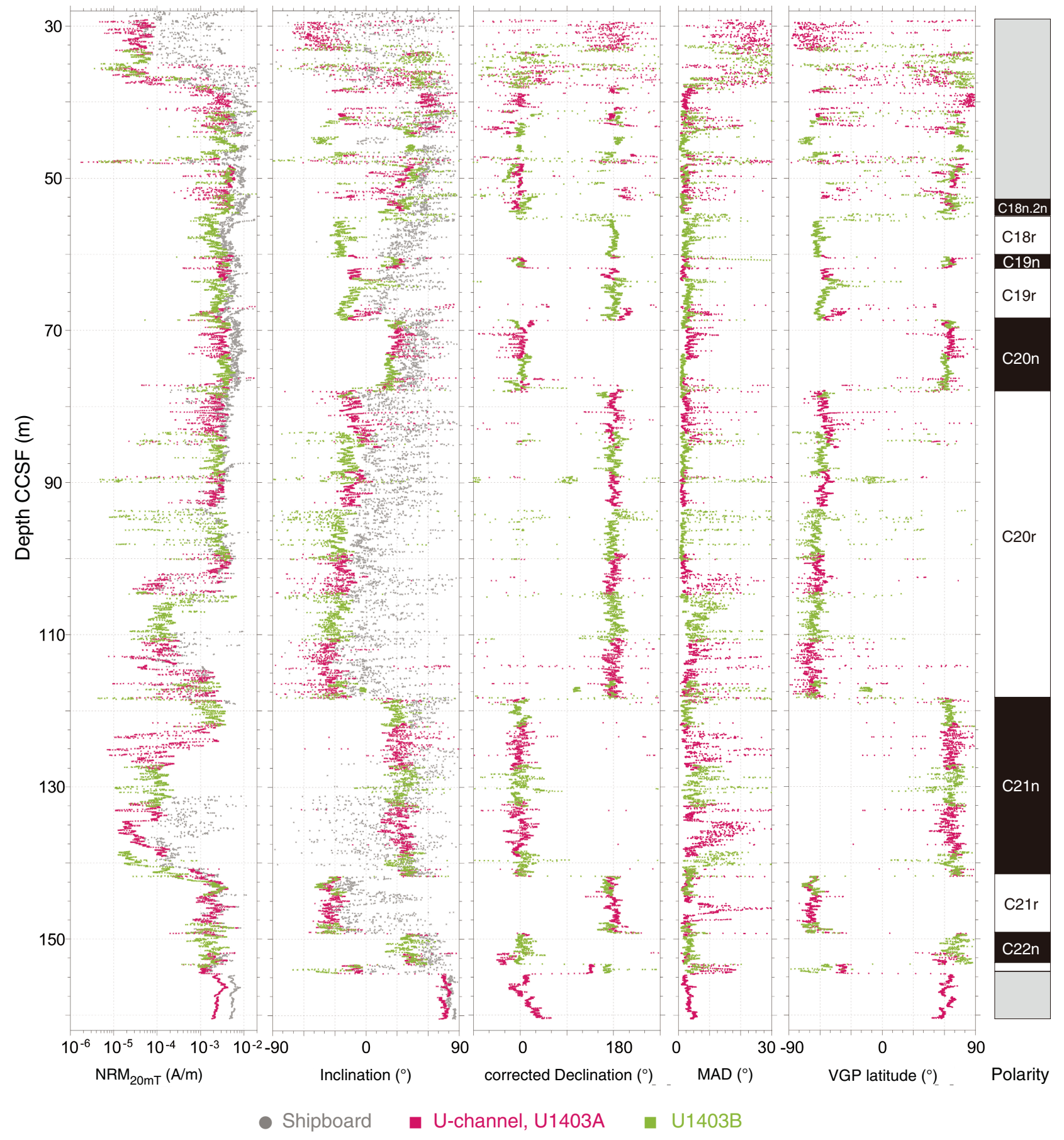


Figure F6. Plots of downhole variation of NRM intensity after $20 \mathrm{mT}$ demagnetization, PCA inclination, corrected PCA declination, maximum angular deviation (MAD) and virtual geomagnetic pole (VGP) latitude for Site U1408. Pink = Hole U1408A; green $=$ Hole U1408B; blue $=$ Hole U1408C. Orange $=$ discrete samples; shaded $=$ shipboard data. Interpreted paleomagnetic polarities and chrons are indicated in the rightmost panel (black $=$ normal, white $=$ reversed, shaded $=$ unidentified $)$.

Site U1408

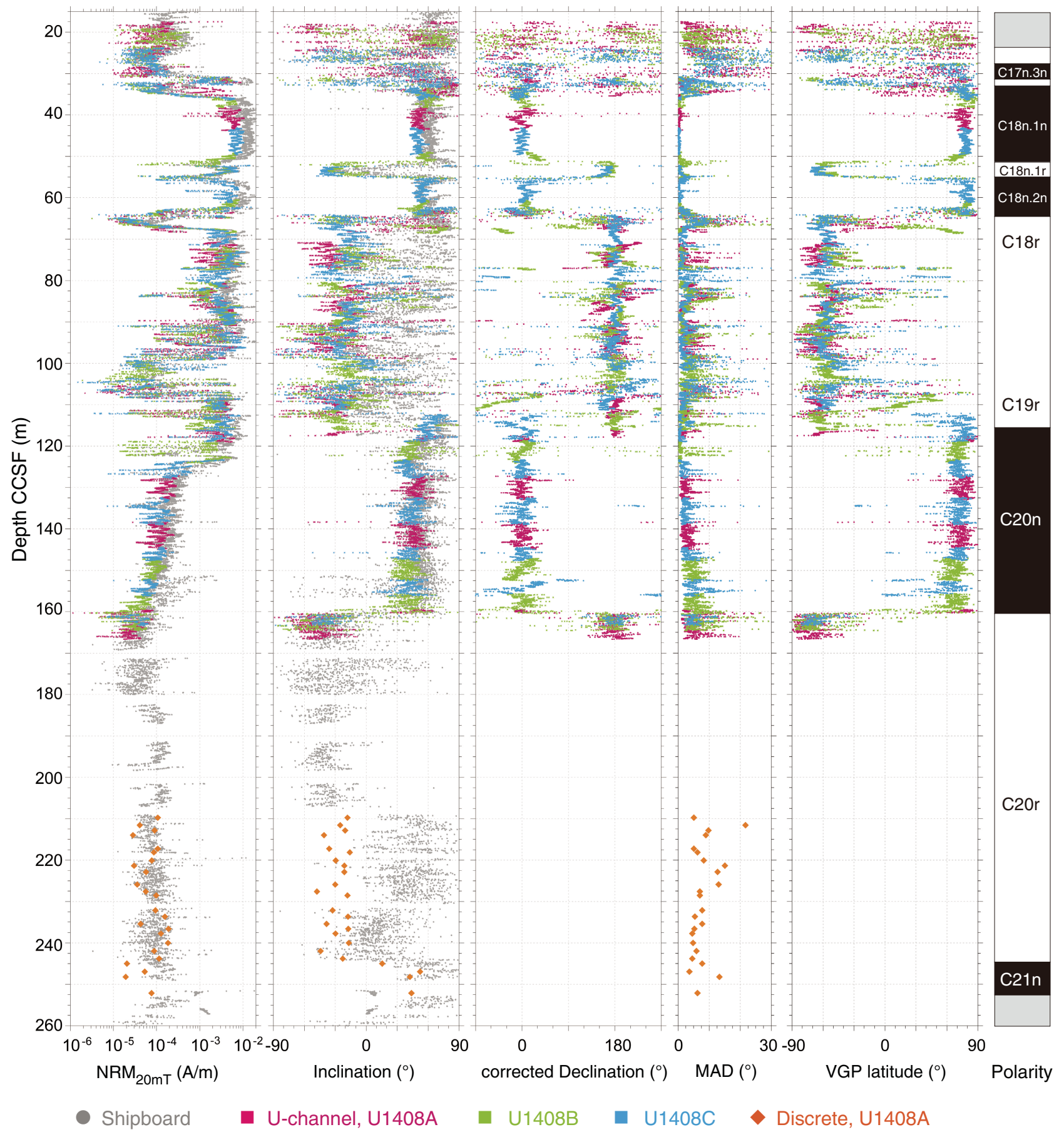


Figure F7. Plots of downhole variation of NRM intensity after $20 \mathrm{mT}$ demagnetization, PCA inclination, corrected PCA declination, maximum angular deviation (MAD) and virtual geomagnetic pole (VGP) latitude for Site U1409. Pink = Hole U1409A; green $=$ Hole U1409B; blue $=$ Hole U1409C. Shaded $=$ shipboard data . Interpreted paleomagnetic polarities and chrons are indicated in the rightmost panel (black $=$ normal, white $=$ reversed, shaded $=$ unidentified).

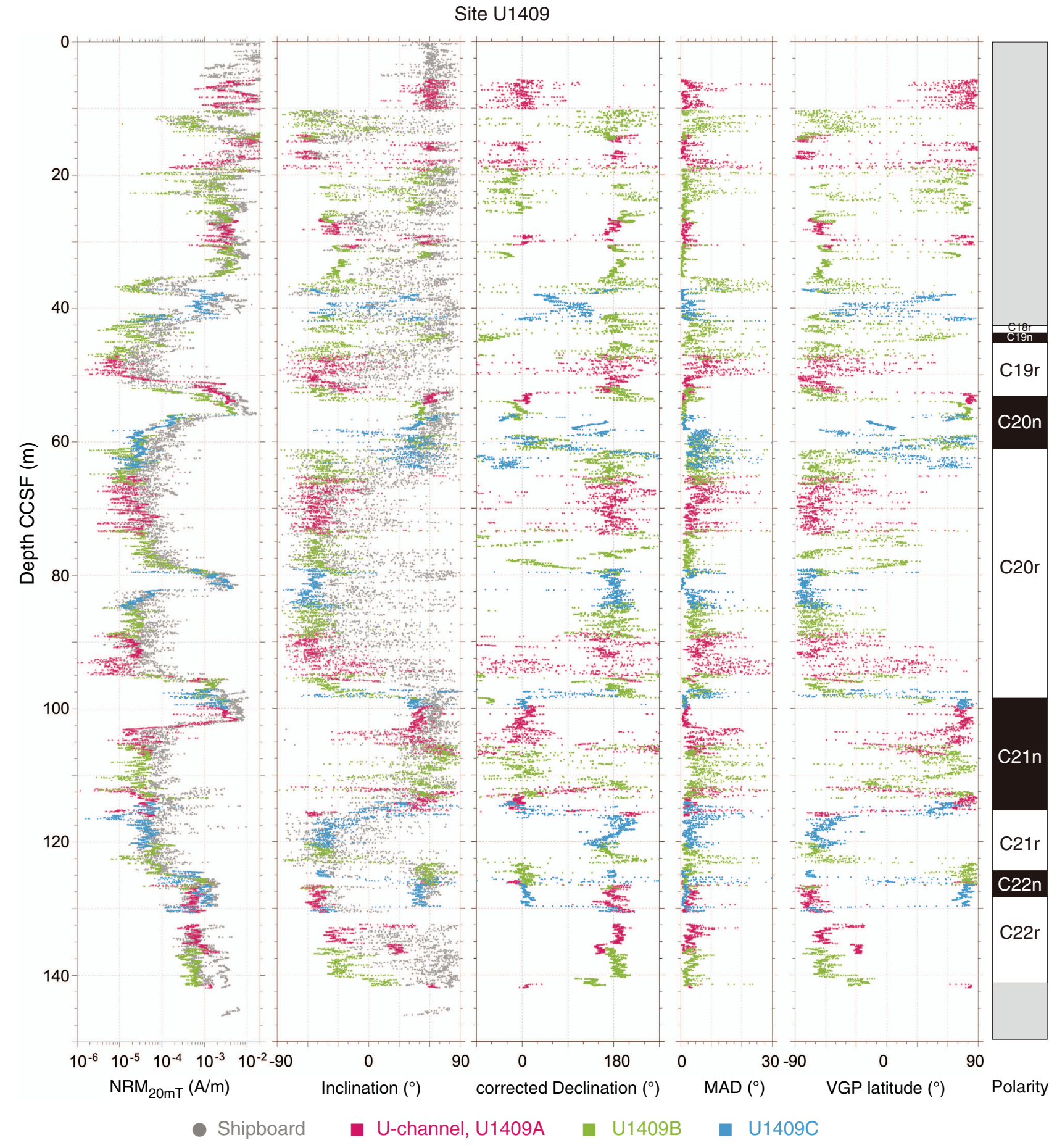


Figure F8. Plots of downhole variation of NRM intensity after $20 \mathrm{mT}$ demagnetization, PCA inclination, corrected PCA declination, maximum angular deviation (MAD) and virtual geomagnetic pole (VGP) latitude for Site U1410. Pink = Hole U1410A; green $=$ Hole U1410B; blue $=$ Hole U1410C. Shaded $=$ shipboard data . Interpreted paleomagnetic polarities and chrons are indicated in the rightmost panel (black $=$ normal, white $=$ reversed, shaded $=$ unidentified).

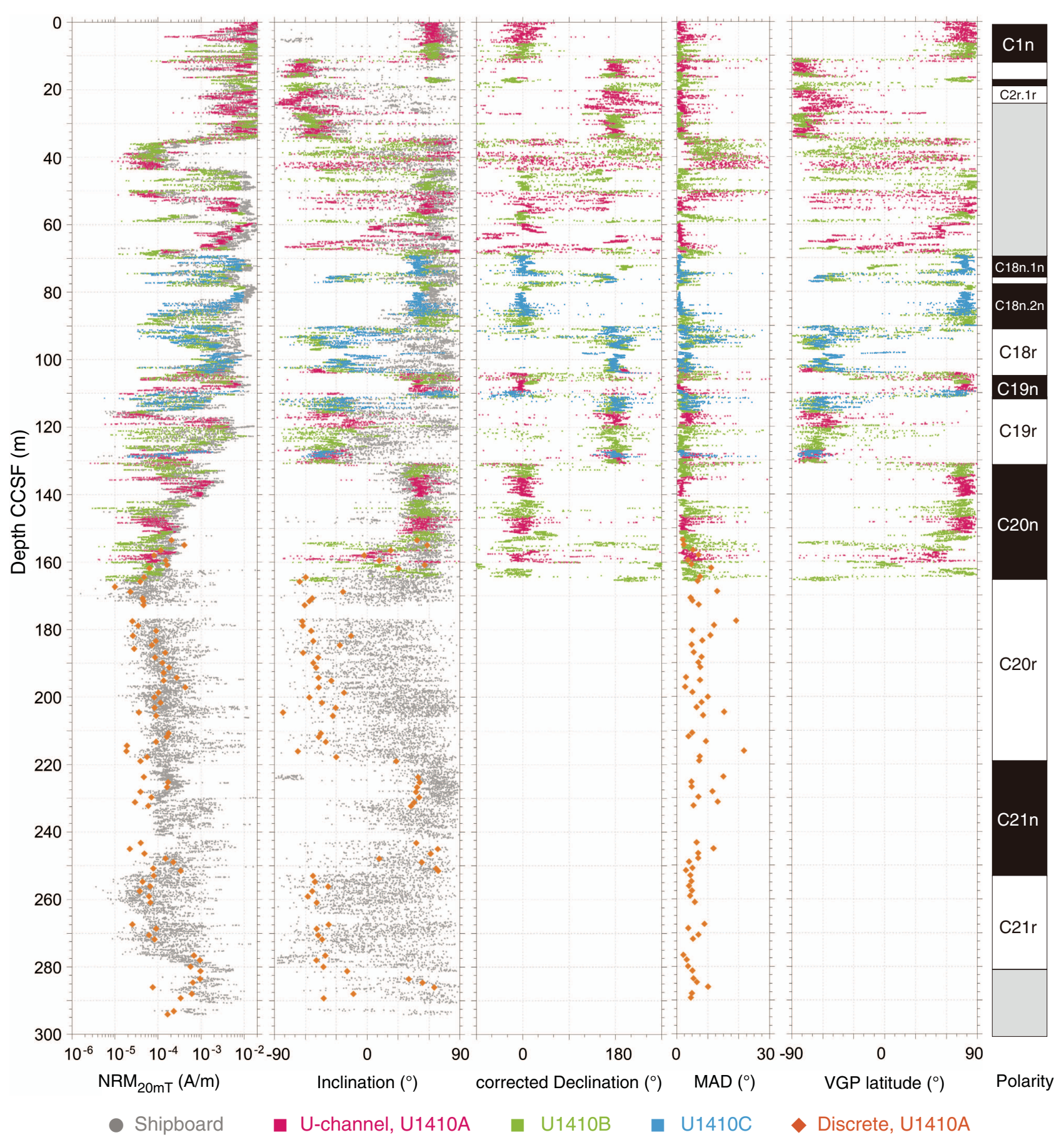


Table T1. Summary of AF demagnetization results for discrete samples from Hole U1408A.

\begin{tabular}{|c|c|c|c|c|c|c|c|}
\hline \multirow{2}{*}{$\begin{array}{l}\text { Core, section, } \\
\text { interval }(\mathrm{cm})\end{array}$} & \multicolumn{2}{|c|}{ Depth $(m)$} & \multirow{2}{*}{$\begin{array}{c}\mathrm{NRM}_{20 \mathrm{mT}} \\
\left(10^{-5} \mathrm{~A} / \mathrm{m}\right)\end{array}$} & \multicolumn{3}{|c|}{ Principal component analysis $\left({ }^{\circ}\right)$} & \multirow{2}{*}{$\begin{array}{l}\text { Range } \\
(\mathrm{mT})\end{array}$} \\
\hline & CSF & CCSF & & Declination & Inclination & MAD & \\
\hline \multicolumn{8}{|l|}{ 342-U1408A- } \\
\hline $21 X-1,75-77$ & 183.66 & 209.74 & 10.6 & 196.4 & -18.1 & 5.1 & $22-50$ \\
\hline $21 \mathrm{X}-2,110-112$ & 185.51 & 211.59 & 4.08 & 202.2 & -25.1 & 21.7 & $20-50$ \\
\hline $21 X-3,86-88$ & 186.77 & 212.85 & 8.96 & -37.7 & -20.2 & 9.8 & $20-50$ \\
\hline $21 X-4,51-53$ & 187.92 & 214.00 & 2.84 & 113.1 & -40.9 & 8.9 & $12-38$ \\
\hline $21 X-6,78-80$ & 191.19 & 217.27 & 10.6 & -64.3 & -35.7 & 5.1 & $18-80$ \\
\hline $21 X-7,28-30$ & 192.09 & 218.17 & 8.62 & 218.7 & -15.7 & 6.2 & $15-45$ \\
\hline $22 \mathrm{X}-1,72-74$ & 189.73 & 220.10 & 7.72 & 40.2 & -29.5 & 8.3 & $15-50$ \\
\hline $22 X-2,72-74$ & 191.03 & 221.40 & 3.00 & 33.1 & -21.1 & 15.1 & $18-45$ \\
\hline $22 X-3,72-74$ & 192.53 & 222.90 & 5.75 & -22.9 & -20.9 & 12.7 & $18-40$ \\
\hline $22 X-5,72-74$ & 195.53 & 225.90 & 3.53 & 140.1 & -29.9 & 13.0 & $20-38$ \\
\hline $22 X-6,88-90$ & 197.21 & 227.58 & 5.60 & -24.3 & -47.8 & 7.0 & $10-55$ \\
\hline $22 X-7,34-36$ & 198.17 & 228.54 & 9.79 & 248.7 & -17.9 & 7.0 & $25-45$ \\
\hline $23 X-1,73-75$ & 199.24 & 232.11 & 9.38 & 154.2 & -32.4 & 7.8 & $12-80$ \\
\hline $23 X-2,77-79$ & 200.78 & 233.65 & 15.5 & 189.1 & -17.6 & 5.4 & $18-40$ \\
\hline $23 X-3,103-105$ & 202.54 & 235.41 & 4.22 & 120.0 & -38.3 & 7.8 & $10-40$ \\
\hline $23 X-4,73-75$ & 203.73 & 236.60 & 18.9 & -77.1 & -17.2 & 5.2 & $15-40$ \\
\hline $23 X-5,37-39$ & 204.88 & 237.75 & 12.6 & 23.8 & -29.7 & 4.6 & $22-50$ \\
\hline $23 X-6,113-115$ & 207.14 & 240.01 & 18.5 & 255.6 & -16.8 & 4.8 & $18-40$ \\
\hline $24 X-1,49-51$ & 208.60 & 241.98 & 8.77 & -56.0 & -44.1 & 5.9 & $10-65$ \\
\hline $24 \mathrm{X}-2,83-85$ & 210.44 & 243.82 & 11.5 & 138.1 & -22.7 & 4.6 & $12-80$ \\
\hline $24 \mathrm{X}-3,48-50$ & 211.59 & 244.97 & 2.07 & 230.5 & 15.6 & 7.8 & $10-35$ \\
\hline 24X-4, 99-101 & 213.60 & 246.98 & 5.31 & 200.0 & 52.3 & 3.6 & $12-35$ \\
\hline $24 X-5,76-78$ & 214.87 & 248.25 & 1.90 & -24.9 & 42.4 & 13.3 & $18-30$ \\
\hline $25 X-1,85-87$ & 218.56 & 252.14 & 7.64 & -63.2 & 44.0 & 6.2 & $20-35$ \\
\hline
\end{tabular}

Depth scales in CCSF are based on the splice table provided by Boulila et al. (2018). MAD = maximum angular deviation. 
Table T2. Summary of AF demagnetization results for discrete samples from Hole U1410A (Continued on next page).

\begin{tabular}{|c|c|c|c|c|c|c|c|}
\hline \multirow{2}{*}{$\begin{array}{l}\text { Core, section, } \\
\text { interval }(\mathrm{cm})\end{array}$} & \multicolumn{2}{|c|}{ Depth (m) } & \multirow{2}{*}{$\begin{array}{c}\mathrm{NRM}_{20 \mathrm{mT}} \\
\left(10^{-5} \mathrm{~A} / \mathrm{m}\right)\end{array}$} & \multicolumn{3}{|c|}{ Principal component analysis $\left({ }^{\circ}\right)$} & \multirow{2}{*}{$\begin{array}{l}\text { Range } \\
(\mathrm{mT})\end{array}$} \\
\hline & CSF & CCSF & & Declination & Inclination & MAD & \\
\hline \multicolumn{8}{|l|}{ 342-U1410A- } \\
\hline $16 \mathrm{H}-1,75-77$ & 142.26 & 153.50 & 20.5 & 60.4 & 48.5 & 2.0 & $12-70$ \\
\hline $16 \mathrm{H}-2,75-77$ & 143.76 & 155.00 & 40.9 & 31.8 & 58.3 & 2.3 & $18-80$ \\
\hline $16 \mathrm{H}-4,75-77$ & 146.76 & 158.00 & 8.09 & -24.6 & -2.9 & 7.0 & $20-80$ \\
\hline $16 \mathrm{H}-5,75-77$ & 148.26 & 159.50 & 15.1 & -30.0 & 11.9 & 3.6 & $18-55$ \\
\hline $16 \mathrm{H}-6,60-62$ & 149.61 & 160.85 & 16.3 & 70.2 & 55.9 & 4.7 & $18-65$ \\
\hline $16 \mathrm{H}-7,25-27$ & 150.62 & 161.86 & 6.30 & 110.0 & 30.5 & 11.1 & $20-80$ \\
\hline $17 X-1,91-93$ & 151.92 & 164.51 & 4.75 & 167.4 & -59.5 & 7.5 & $22-55$ \\
\hline $17 X-2,70-72$ & 153.21 & 165.80 & 3.86 & 171.5 & -65.5 & 6.7 & $10-65$ \\
\hline $17 X-3,75-77$ & 154.76 & 167.35 & 1.00 & - & - & - & - \\
\hline $17 X-4,75-77$ & 156.26 & 168.85 & 2.28 & 26.9 & -23.3 & 13.1 & $10-80$ \\
\hline $17 X-5,119-121$ & 158.20 & 170.79 & 4.36 & -4.7 & -53.3 & 4.5 & $18-50$ \\
\hline $17 X-6,52-54$ & 159.03 & 171.62 & 4.67 & -20.4 & -55.9 & 5.1 & $18-80$ \\
\hline $17 X-7,23-25$ & 160.15 & 172.74 & 4.64 & 205.1 & -60.5 & 7.1 & $18-80$ \\
\hline $18 X-1,90-92$ & 161.51 & 177.51 & 2.5 & 245.7 & -62.9 & 19.2 & $20-80$ \\
\hline $18 X-2,75-77$ & 162.86 & 178.86 & 3.45 & 175.2 & -62.3 & 12.0 & $18-60$ \\
\hline $18 X-3,75-77$ & 164.36 & 180.36 & 9.1 & 185.6 & -54.2 & 5.1 & $12-70$ \\
\hline $18 X-4,75-77$ & 165.86 & 181.86 & 2.6 & 111.5 & -15.4 & 10.9 & $15-50$ \\
\hline $18 X-5,75-77$ & 167.36 & 183.36 & 8.8 & 182.1 & -52.1 & 8.2 & $18-60$ \\
\hline $18 X-6,55-57$ & 168.66 & 184.66 & 7.25 & 31.9 & -26.3 & 4.8 & $18-55$ \\
\hline $18 X-7,30-32$ & 169.73 & 185.73 & 2.8 & - & - & - & - \\
\hline $19 X-1,83-85$ & 171.04 & 186.91 & 14.7 & 294.0 & -62.1 & 5.5 & $22-55$ \\
\hline $19 X-2,75-77$ & 172.46 & 188.33 & 8.48 & 200.2 & -47.4 & 8.0 & $22-80$ \\
\hline $19 X-3,77-79$ & 173.98 & 189.85 & 12.7 & 208.9 & -52.3 & 7.0 & $20-80$ \\
\hline $19 X-5,75-77$ & 176.96 & 192.83 & 13.5 & - & - & - & - \\
\hline $19 X-6,75-77$ & 178.46 & 194.33 & 27.3 & 31.8 & -47.2 & 2.9 & $18-80$ \\
\hline $19 X-7,25-27$ & 179.27 & 195.14 & 13.5 & 205.7 & -34.6 & 7.5 & $18-55$ \\
\hline $20 X-1,75-77$ & 180.56 & 197.18 & 43.1 & 13.5 & -47.0 & 2.7 & $22-80$ \\
\hline $20 \mathrm{X}-2,81-83$ & 182.12 & 198.74 & 10.2 & -67.2 & -22.4 & 5.1 & $18-70$ \\
\hline $20 X-3,68-70$ & 183.49 & 200.11 & 8.19 & 39.9 & -56.0 & 10.0 & $18-70$ \\
\hline $20 X-5,77-79$ & 186.58 & 203.20 & 8.23 & 43.7 & -30.5 & 6.4 & $22-70$ \\
\hline $20 X-6,65-67$ & 187.96 & 204.58 & 3.59 & 219.2 & -81.5 & 15.3 & $18-80$ \\
\hline $20 X-7,31-33$ & 188.93 & 205.55 & 9.10 & 228.6 & -33.4 & 8.5 & $22-65$ \\
\hline $21 X-1,39-41$ & 189.80 & 210.81 & 17.6 & -42.3 & -44.9 & 5.0 & $18-80$ \\
\hline $21 X-2,77-79$ & 190.82 & 211.83 & 15.9 & 207.2 & -46.8 & 3.8 & $22-70$ \\
\hline $21 X-3,70-72$ & 192.25 & 213.26 & 9.04 & 269.7 & -40.2 & 9.4 & $15-65$ \\
\hline $21 X-4,42-44$ & 193.43 & 214.44 & 1.89 & - & - & - & - \\
\hline $21 X-5,65-67$ & 195.03 & 216.04 & 1.86 & 156.6 & -67.2 & 21.7 & $18-65$ \\
\hline $21 X-6,90-92$ & 196.78 & 217.79 & 5.56 & 36.1 & -30.0 & 7.4 & $12-60$ \\
\hline $21 X-7,62-64$ & 198.00 & 219.01 & 3.94 & 174.6 & 28.4 & 7.3 & $15-60$ \\
\hline $22 X-1,74-76$ & 199.75 & 223.75 & 4.69 & -24.6 & 49.5 & 15.1 & $22-50$ \\
\hline $22 X-2,76-78$ & 201.27 & 225.27 & 17.1 & 20.6 & 50.9 & 4.7 & $25-80$ \\
\hline $22 X-3,70-72$ & 202.71 & 226.71 & 16.3 & 22.9 & 48.6 & 4.8 & $20-60$ \\
\hline $22 X-4,67-69$ & 204.18 & 228.18 & 3.93 & 213.0 & 47.6 & 11.5 & $18-65$ \\
\hline $22 X-5,74-76$ & 205.75 & 229.75 & 7.08 & 121.7 & 50.6 & 7.0 & $10-80$ \\
\hline $22 X-6,72-74$ & 207.23 & 231.23 & 2.93 & -51.5 & 45.6 & 13.2 & $15-60$ \\
\hline $22 X-7,26-28$ & 208.27 & 232.27 & 5.89 & 114.9 & 42.9 & 5.4 & $10-65$ \\
\hline $23 X-1,83-85$ & 209.34 & 243.29 & 3.99 & 57.5 & 47.8 & 6.4 & $12-80$ \\
\hline $23 X-2,109-111$ & 211.10 & 245.05 & 2.21 & 209.6 & 68.6 & 11.9 & $18-50$ \\
\hline $23 X-3,98-100$ & 212.49 & 246.44 & 4.83 & 146.8 & 61.8 & 6.9 & $10-70$ \\
\hline $23 X-4,93-95$ & 213.94 & 247.89 & 15.0 & 84.3 & 11.9 & 6.9 & $10-70$ \\
\hline $23 X-5,47-49$ & 214.98 & 248.93 & 22.5 & 158.2 & 53.1 & 4.0 & $20-65$ \\
\hline $23 X-6,92-94$ & 216.93 & 250.88 & 7.78 & 148.3 & 66.9 & 5.1 & $15-65$ \\
\hline $23 X-7,9-11$ & 217.60 & 251.55 & 33.6 & 66.4 & 69.1 & 3.0 & $15-70$ \\
\hline $24 X-1,51-53$ & 218.52 & 252.99 & 7.90 & 67.9 & -52.5 & 4.4 & $30-70$ \\
\hline $24 X-2,75-77$ & 220.26 & 254.73 & 4.35 & 252.4 & -50.8 & 4.7 & $20-65$ \\
\hline $24 X-3,73-75$ & 221.74 & 256.21 & 6.59 & 63.6 & -37.7 & 4.0 & $12-70$ \\
\hline $24 X-4,52-54$ & 223.03 & 257.50 & 3.76 & 11.4 & -53.2 & 4.9 & $18-70$ \\
\hline $24 X-5,60-62$ & 224.61 & 259.08 & 6.15 & 10.9 & -57.7 & 4.3 & $18-60$ \\
\hline $24 X-6,97-99$ & 226.48 & 260.95 & 6.64 & 256.7 & -48.8 & 5.8 & $18-80$ \\
\hline $25 X-1,67-69$ & 228.18 & 267.44 & 2.54 & 138.0 & -37.4 & 8.9 & $18-65$ \\
\hline $25 X-2,39-41$ & 229.40 & 268.66 & 9.09 & 194.5 & -48.9 & 3.7 & $18-80$ \\
\hline $25 X-3,84-86$ & 231.35 & 270.61 & 6.14 & 43.2 & -47.4 & 7.0 & $18-60$ \\
\hline $25 X-4,58-60$ & 232.59 & 271.85 & 8.38 & 130.8 & -43.5 & 5.2 & $12-80$ \\
\hline $26 X-4,53-55$ & 242.14 & 278.00 & 94.0 & 131.2 & -49.0 & 3.2 & $20-65$ \\
\hline $26 X-5,95-97$ & 244.06 & 279.92 & 56.5 & 195.8 & -42.4 & 3.6 & $25-55$ \\
\hline $26 X-6,77-79$ & 245.38 & 281.24 & 96.0 & 69.0 & -19.3 & 5.1 & $18-32$ \\
\hline
\end{tabular}


Table T2 (continued).

\begin{tabular}{|c|c|c|c|c|c|c|c|}
\hline \multirow{2}{*}{$\begin{array}{l}\text { Core, section, } \\
\text { interval }(\mathrm{cm})\end{array}$} & \multicolumn{2}{|c|}{ Depth (m) } & \multirow{2}{*}{$\begin{array}{l}\mathrm{NRM}_{20 \mathrm{mT}} \\
\left(10^{-5} \mathrm{~A} / \mathrm{m}\right)\end{array}$} & \multicolumn{3}{|c|}{ Principal component analysis $\left({ }^{\circ}\right)$} & \multirow{2}{*}{$\begin{array}{c}\text { Range } \\
(\mathrm{mT})\end{array}$} \\
\hline & CSF & CCSF & & Declination & Inclination & $\overline{M A D}$ & \\
\hline $27 X-1,104-106$ & 247.75 & 283.61 & 95.1 & 80.9 & 40.5 & 5.4 & $12-45$ \\
\hline $27 X-2,54-56$ & 248.75 & 284.61 & 63.9 & 105.6 & 53.9 & 6.5 & $20-40$ \\
\hline $27 X-3,46-48$ & 250.17 & 286.03 & 7.51 & 22.4 & 65.3 & 10.1 & $12-30$ \\
\hline $27 X-4,93-95$ & 252.14 & 288.00 & 60.6 & -7.6 & -13.2 & 4.9 & $20-70$ \\
\hline $27 X-5,74-76$ & 253.45 & 289.31 & 33.5 & 75.5 & -42.1 & 4.6 & $12-40$ \\
\hline $28 \mathrm{X}-1,96-98$ & 257.27 & 293.13 & 23.3 & - & - & - & - \\
\hline $28 X-2,41-43$ & 258.22 & 294.08 & 16.7 & - & - & - & - \\
\hline
\end{tabular}

Depth scales in CCSF are based on the splice table provided by Boulila et al. (2018). MAD = maximum angular deviation. 
Table T3. Core orientation data.

Core Hole U1403A $\left(^{\circ}\right)$ Hole U1403B $\left(^{\circ}\right)$ Hole U1408A $\left(^{\circ}\right)$ Hole U1408B $\left(^{\circ}\right)$ Hole U1408C $\left(^{\circ}\right)$ Hole U1409A $\left(^{\circ}\right)$ Hole U1409B $\left(^{\circ}\right)$ Hole U1409C $\left(^{\circ}\right)$ Hole U1410A $\left(^{\circ}\right)$ Hole U1410B $\left(^{\circ}\right)$ Hole U1410C $\left(^{\circ}\right)$ MTF Dec Sum MTF Dec Sum MTF Dec Sum MTF Dec Sum MTF Dec Sum MTF Dec Sum MTF Dec Sum MTF Dec Sum MTF Dec Sum MTF Dec Sum MTF Dec Sum

\begin{tabular}{|c|c|c|c|c|c|c|c|c|c|c|c|c|c|c|c|c|c|c|c|c|c|c|c|c|c|c|c|c|c|}
\hline 1 & & & & & & & 100 & & & 185 & & & & & & -43 & & & -59 & & & & 129 & 256 & 25 & 158 & & & \\
\hline 2 & & & & & & & 116 & & & -64 & & & & & & 74 & -70 & 4 & -31 & 54 & 23 & & 198 & 202 & 40 & 140 & 261 & 41 & \\
\hline 3 & & & & & & & -61 & 62 & 1 & 36 & -46 & -10 & & & & 183 & 213 & 36 & 143 & 237 & 20 & & -49 & 65 & 16 & 158 & 239 & 37 & \\
\hline 4 & 216 & 126 & -18 & & & & -66 & 217 & 151 & & & & & 266 & & 23 & 19 & 42 & 120 & -62 & 58 & & 34 & -6 & 29 & 119 & -89 & 30 & \\
\hline 5 & 21 & -9 & 12 & 250 & 119 & 9 & 103 & 250 & -7 & 7 & -23 & -16 & & -43 & & -19 & & & 77 & -51 & 26 & 124 & -87 & 100 & 13 & 118 & -61 & 57 & \\
\hline 6 & 135 & 221 & -4 & 173 & 201 & 14 & 106 & & & & & & 221 & 155 & 16 & -30 & 34 & 4 & 109 & 247 & -4 & & -18 & 56 & 38 & -1 & 41 & 40 & \\
\hline 7 & 18 & 6 & 24 & 140 & 195 & -25 & 251 & 77 & -32 & & 43 & & 257 & 99 & -4 & 203 & & & -77 & 93 & 16 & 69 & 228 & 57 & -75 & 216 & 155 & 11 & \\
\hline 8 & 250 & -49 & 201 & 125 & 211 & -24 & 185 & 153 & -22 & & 89 & & -11 & -2 & -13 & 67 & -37 & 30 & 242 & 63 & -55 & & 211 & & & 42 & -19 & 23 & 143 \\
\hline 9 & 133 & 195 & -32 & 189 & 144 & -27 & 118 & 263 & 21 & & 234 & & & 211 & & 152 & & & 198 & 134 & -28 & 88 & 144 & & & -67 & 88 & 21 & 230 \\
\hline 10 & 232 & 100 & -28 & & 198 & & 252 & 83 & -25 & & -27 & & & -8 & & 117 & 260 & 17 & 122 & 267 & 29 & & -72 & & & 39 & -37 & 2 & 231 \\
\hline 11 & 82 & 230 & -48 & & 57 & & 263 & 58 & -39 & & 159 & & & 137 & & -60 & 101 & 41 & -79 & 7 & -72 & 176 & 159 & 190 & -12 & 225 & 125 & -10 & -6 \\
\hline 12 & 110 & 259 & 9 & & -57 & & -45 & 22 & -23 & & 82 & & & -19 & & 256 & 64 & -40 & -37 & 46 & 9 & 15 & -43 & 11 & -32 & 133 & 247 & 20 & 154 \\
\hline 13 & 202 & 143 & -15 & & 167 & & 219 & 133 & -8 & & -54 & & & 195 & & 84 & -74 & 10 & 55 & -32 & 23 & 215 & 52 & -33 & 19 & -4 & 11 & 7 & 70 \\
\hline 14 & 147 & 189 & -24 & & -45 & & -78 & 85 & 7 & & 262 & & & 103 & & -6 & 8 & 2 & 230 & & & & 123 & 260 & 23 & 259 & 98 & -3 & \\
\hline 15 & 17 & -11 & 6 & & -52 & & 80 & & & & & & & -73 & & 168 & & & & & & & 7 & 28 & 35 & 41 & -11 & 30 & \\
\hline 16 & 259 & 132 & 31 & & 71 & & 100 & 255 & -5 & & & & & 267 & & -43 & & & & & & & -30 & -24 & -54 & 104 & -83 & 21 & \\
\hline 17 & & & & & & & 224 & & & & -80 & & & 12 & & & & & & & & & & & & 166 & 211 & 17 & \\
\hline 18 & & & & & & & -85 & & & & 23 & & & 183 & & & & & & & & & & & & 96 & -65 & 31 & \\
\hline 19 & & & & & & & -69 & & & & & & & & & & & & & & & & & & & & & & \\
\hline 20 & & & & & & & 156 & & & & & & & & & & & & & & & & & & & & & & \\
\hline
\end{tabular}

MTF = magnetic tool face orientation from geomagnetic north $\left(-90^{\circ} \sim 270^{\circ}\right)$. Dec $=$ mean declination in the sample coordinate $\left(-90^{\circ} \sim 270^{\circ}\right)$. Sum $=\mathrm{MTF}+$ Dec $\left(-90^{\circ} \sim 270^{\circ}\right)$. 
Table T4. Magnetostratigraphic tie points, Site U1403.

\begin{tabular}{|c|c|c|c|c|c|c|c|}
\hline \multirow{3}{*}{$\begin{array}{l}\text { Chron } \\
\text { boundary }\end{array}$} & \multirow{3}{*}{$\begin{array}{c}\text { Age } \\
\text { GTS2012(Ma) }\end{array}$} & \multicolumn{6}{|c|}{ Depth $(\mathrm{m})$} \\
\hline & & \multicolumn{2}{|c|}{ Hole U1403A } & \multicolumn{2}{|c|}{ Hole U1403B } & \multirow{2}{*}{$\begin{array}{c}\text { Average } \\
\text { CCSF }\end{array}$} & \multirow{2}{*}{$\begin{array}{l}\text { StDev } \\
\text { CCSF }\end{array}$} \\
\hline & & CSF* & CCSF & CSF* $^{*}$ & CCSF & & \\
\hline $\mathrm{C} 18 n .2 n / C 18 r$ & 40.145 & - & - & $\begin{array}{l}47.57 \\
(-0.26)\end{array}$ & 55.17 & 55.17 & - \\
\hline $\mathrm{C} 18 \mathrm{r} / \mathrm{C} 19 \mathrm{n}$ & 41.154 & $\begin{array}{l}58.70 \\
(+0.03)\end{array}$ & 60.48 & $\begin{array}{c}56.53 \\
(+3.79)\end{array}$ & 60.34 & 60.41 & 0.10 \\
\hline $\mathrm{C} 19 n / C 19 r$ & 41.390 & $\begin{array}{l}60.06 \\
(-0.03)\end{array}$ & 61.84 & - & - & 61.84 & - \\
\hline C19r/C20n & 42.301 & $\begin{array}{l}64.91 \\
(-0.05)\end{array}$ & 68.67 & $\begin{array}{l}60.27 \\
(-0.02)\end{array}$ & 68.65 & 68.66 & 0.01 \\
\hline $\mathrm{C} 20 \mathrm{n} / \mathrm{C} 20 \mathrm{r}$ & 43.432 & $\begin{array}{l}73.78 \\
(-0.21)\end{array}$ & 77.97 & $\begin{array}{c}69.40 \\
(+0.18)\end{array}$ & 78.08 & 78.02 & 0.07 \\
\hline $\mathrm{C} 20 \mathrm{r} / \mathrm{C} 21 \mathrm{n}$ & 45.724 & $\begin{array}{l}109.64 \\
(-0.04)\end{array}$ & 118.30 & $\begin{array}{l}106.85 \\
(+0.05)\end{array}$ & 118.47 & 118.39 & 0.12 \\
\hline $\mathrm{C} 21 \mathrm{n} / \mathrm{C} 21 \mathrm{r}$ & 47.349 & $\begin{array}{l}130.36 \\
(+0.02)\end{array}$ & 141.78 & $\begin{array}{l}126.00 \\
(-0.04)\end{array}$ & 141.75 & 141.76 & 0.02 \\
\hline$C 21 r / C 22 n$ & 48.566 & $\begin{array}{l}137.84 \\
(+0.00)\end{array}$ & 149.26 & $\begin{array}{l}132.90 \\
(+0.00)\end{array}$ & 149.33 & 149.29 & 0.05 \\
\hline $\mathrm{C} 22 \mathrm{n} / \mathrm{C} 22 \mathrm{r}$ & 49.344 & $\begin{array}{l}140.43 \\
(+0.13)\end{array}$ & 153.39 & $\begin{array}{l}136.89 \\
(-0.02)\end{array}$ & 153.32 & 153.36 & 0.05 \\
\hline
\end{tabular}

Chron boundary ages are referred from Ogg (2012). ${ }^{*}=$ values in parentheses are differences from the shipboard data. Depth scales in CCSF are based on the splice table provided in the "Site U1403" chapter (Norris et al., 2014c).

Table T5. Magnetostratigraphic tie points, Site U1408.

\begin{tabular}{|c|c|c|c|c|c|c|c|c|c|}
\hline \multirow{3}{*}{$\begin{array}{l}\text { Chron } \\
\text { boundary }\end{array}$} & \multirow{3}{*}{$\begin{array}{c}\text { Age } \\
\text { GTS2012 (Ma) }\end{array}$} & \multicolumn{8}{|c|}{ Depth (m) } \\
\hline & & \multicolumn{2}{|c|}{ Hole U1408A } & \multicolumn{2}{|c|}{ Hole U1408B } & \multicolumn{2}{|c|}{ Hole U1408C } & \multirow{2}{*}{$\begin{array}{l}\text { Average } \\
\text { CCSF }\end{array}$} & \multirow{2}{*}{$\begin{array}{l}\text { StDev } \\
\text { CCSF }\end{array}$} \\
\hline & & $\mathrm{CSF}^{*}$ & CCSF & $\mathrm{CSF}^{*}$ & CCSF & $\mathrm{CSF}^{*}$ & CCSF & & \\
\hline C17n.2r/C17n.3n & 38.159 & - & - & - & - & 24.96 & 27.26 & 27.26 & - \\
\hline $\mathrm{C} 17 \mathrm{n} .3 \mathrm{n} / \mathrm{C} 17 \mathrm{r}$ & 38.333 & - & - & - & - & 28.91 & 31.21 & 31.21 & - \\
\hline C17r/C18n.1n & 38.615 & - & - & $\begin{array}{c}32.40 \\
(+0.41)\end{array}$ & 32.95 & 30.81 & 32.51 & 32.73 & 0.31 \\
\hline C18n.1n/C18n.1r & 39.627 & - & - & $\begin{array}{l}51.24 \\
(-0.31)\end{array}$ & 51.64 & $\begin{array}{l}49.97 \\
(-0.01)\end{array}$ & 51.64 & 51.64 & 0.00 \\
\hline C18n.1r/C18n.2n & 39.698 & - & - & $\begin{array}{l}54.63 \\
(+0.12)\end{array}$ & 55.03 & $\begin{array}{l}52.66 \\
(-0.37)\end{array}$ & 55.01 & 55.02 & 0.01 \\
\hline $\mathrm{C} 18 n .2 n / C 18 r$ & 40.145 & - & - & 62.70 & 64.45 & $\begin{array}{l}61.21 \\
(-2.14)\end{array}$ & 64.16 & 64.30 & 0.20 \\
\hline $\mathrm{C} 18 \mathrm{r} / \mathrm{C} 19 \mathrm{n}$ & 41.154 & - & - & - & - & - & - & - & - \\
\hline $\mathrm{C} 19 n / \mathrm{C} 19 \mathrm{r}$ & 41.390 & - & - & - & - & - & - & - & - \\
\hline $\mathrm{C} 19 \mathrm{r} / \mathrm{C} 20 \mathrm{n}$ & 42.301 & $\begin{array}{l}101.21 \\
(+0.00)\end{array}$ & 117.52 & $\begin{array}{l}110.35 \\
(+3.73)\end{array}$ & 117.39 & $\begin{array}{c}105.00 \\
(-0.53)\end{array}$ & 111.69 & $117.46^{\dagger}$ & $0.09^{\dagger}$ \\
\hline$C 20 n / C 20 r$ & 43.432 & $\begin{array}{l}138.63 \\
(-0.44)\end{array}$ & 160.43 & $\begin{array}{l}149.84 \\
(-0.04)\end{array}$ & 160.03 & - & - & 160.23 & 0.28 \\
\hline $\mathrm{C} 20 \mathrm{r} / \mathrm{C} 21 \mathrm{n}$ & 45.724 & $\begin{array}{l}211.10 \\
(-1.50)\end{array}$ & 244.48 & - & - & - & - & 244.48 & - \\
\hline
\end{tabular}

Chron boundary ages are referred from Ogg (2012). * = values in parentheses are differences from the shipboard data. Depth scales in CCSF are based on the splice table provided by Boulila et al. (2018). $\dagger=$ Hole U1408C data are excluded because they largely differ from the Hole U1408A and U1408B data. 
Table T6. Magnetostratigraphic tie points, Site U1409.

\begin{tabular}{|c|c|c|c|c|c|c|c|c|c|}
\hline \multirow{3}{*}{$\begin{array}{c}\text { Chron } \\
\text { boundary }\end{array}$} & \multirow{3}{*}{$\begin{array}{c}\text { Age } \\
\text { GTS2012 (Ma) }\end{array}$} & \multicolumn{8}{|c|}{ Depth (m) } \\
\hline & & \multicolumn{2}{|c|}{ Hole U1409A } & \multicolumn{2}{|c|}{ Hole U1409B } & \multicolumn{2}{|c|}{ Hole U1409C } & \multirow{2}{*}{$\begin{array}{c}\text { Average } \\
\text { CCSF }\end{array}$} & \multirow{2}{*}{$\begin{array}{l}\text { StDe } \\
\text { CCSF }\end{array}$} \\
\hline & & $\mathrm{CSF}^{*}$ & CCSF & $\mathrm{CSF}^{*}$ & CCSF & $\mathrm{CSF}^{*}$ & CCSF & & \\
\hline $\mathrm{C} 18 \mathrm{r} / \mathrm{C} 19 \mathrm{n}$ & 41.154 & - & - & 41.36 & 43.77 & - & - & 43.77 & - \\
\hline $\mathrm{C} 19 n / \mathrm{C} 19 \mathrm{r}$ & 41.390 & - & - & 42.57 & 44.98 & - & - & 44.98 & - \\
\hline C19r/C20n & 42.301 & $\begin{array}{c}46.26 \\
(+0.01)\end{array}$ & 52.61 & $\begin{array}{c}51.43 \\
(+0.51)\end{array}$ & 53.95 & - & - & 53.28 & 0.95 \\
\hline $\mathrm{C} 20 \mathrm{n} / \mathrm{C} 20 \mathrm{r}$ & 43.432 & - & - & $\begin{array}{c}58.09 \\
(+0.30)\end{array}$ & 61.27 & - & - & 61.27 & - \\
\hline $\mathrm{C} 20 \mathrm{r} / \mathrm{C} 21 \mathrm{n}$ & 45.724 & - & - & $\begin{array}{c}88.09 \\
(+0.66)\end{array}$ & 98.52 & $\begin{array}{c}89.08 \\
(+4.77)\end{array}$ & 97.29 & 97.90 & 0.87 \\
\hline $\mathrm{C} 21 \mathrm{n} / \mathrm{C} 21 \mathrm{r}$ & 47.349 & $\begin{array}{c}101.27 \\
(-0.08)\end{array}$ & 115.40 & - & - & $\begin{array}{c}100.97 \\
(-0.14)\end{array}$ & 116.12 & 115.76 & 0.51 \\
\hline$C 21 r / C 22 n$ & 48.566 & - & - & $\begin{array}{l}108.78 \\
(-0.04)\end{array}$ & 123.26 & $\begin{array}{l}111.22 \\
(+0.32)\end{array}$ & 125.74 & 124.50 & 1.75 \\
\hline $\mathrm{C} 22 \mathrm{n} / \mathrm{C} 22 \mathrm{r}$ & 49.344 & $\begin{array}{c}110.91 \\
(-0.17)\end{array}$ & 126.53 & - & - & $\begin{array}{c}115.24 \\
(+0.03)\end{array}$ & 129.76 & 128.14 & 2.28 \\
\hline
\end{tabular}

Chron boundary ages are referred from Ogg (2012). ${ }^{*}=$ values in parentheses are differences from the shipboard data. Depth scales in CCSF are based on the splice table provided by Hull et al. (2017).

Table T7. Magnetostratigraphic tie points, Site U1410.

\begin{tabular}{|c|c|c|c|c|c|c|c|c|c|}
\hline \multirow{3}{*}{$\begin{array}{l}\text { Chron } \\
\text { boundary }\end{array}$} & \multirow[b]{3}{*}{ Age GTS2012 (Ma) } & \multicolumn{8}{|c|}{ Depth (m) } \\
\hline & & \multicolumn{2}{|c|}{ Hole U1410A } & \multicolumn{2}{|c|}{ Hole U1410B } & \multicolumn{2}{|c|}{ Hole U1410C } & \multirow{2}{*}{$\begin{array}{l}\text { Average } \\
\text { CCSF }\end{array}$} & \multirow{2}{*}{$\begin{array}{l}\text { StDev } \\
\text { CCSF }\end{array}$} \\
\hline & & $\mathrm{CSF}^{*}$ & CCSF & $\mathrm{CSF}^{*}$ & CCSF & CSF* & CCSF & & \\
\hline $\begin{array}{l}\text { C1n (Brunhes)/ } \\
\text { C1r.1r (Matuyama) }\end{array}$ & 0.781 & $\begin{array}{c}11.54 \\
(+0.04)\end{array}$ & 10.74 & $\begin{array}{c}9.96 \\
(-0.02)\end{array}$ & 10.91 & - & - & 10.83 & 0.12 \\
\hline $\begin{array}{l}\text { C1r.3r (Matuyama)/ } \\
\text { C2n (Olduvai) }\end{array}$ & 1.778 & $\begin{array}{l}17.24 \\
(+0.11)\end{array}$ & 16.44 & $\begin{array}{l}15.90 \\
(+0.07)\end{array}$ & 16.45 & - & - & 16.44 & 0.01 \\
\hline $\begin{array}{l}\text { C2n (Olduvai)/ } \\
\text { C2r.1r (Matuyama) }\end{array}$ & 1.945 & - & - & 17.43 & 17.98 & - & - & 17.98 & - \\
\hline $\mathrm{C} 18 n .1 \mathrm{n} / \mathrm{C} 18 \mathrm{n} .1 \mathrm{r}$ & 39.627 & - & - & $\begin{array}{l}69.70 \\
(+0.50)\end{array}$ & 74.83 & $\begin{array}{l}71.08 \\
(-0.32)\end{array}$ & 74.97 & 74.90 & 0.10 \\
\hline $\mathrm{C} 18 n .1 \mathrm{r} / \mathrm{C} 18 \mathrm{n} .2 \mathrm{n}$ & 39.698 & - & - & $\begin{array}{l}71.85 \\
(-1.07)\end{array}$ & 76.98 & 73.36 & 77.25 & 77.11 & 0.19 \\
\hline $\mathrm{C} 18 n \cdot 2 n / C 18 r$ & 40.145 & - & - & $\begin{array}{l}83.60 \\
(-0.21)\end{array}$ & 90.17 & - & - & 90.17 & - \\
\hline $\mathrm{C} 18 \mathrm{r} / \mathrm{C} 19 \mathrm{n}$ & 41.154 & $\begin{array}{l}96.72 \\
(-0.08)\end{array}$ & 103.95 & $\begin{array}{r}96.39 \\
(+0.99)\end{array}$ & 103.90 & - & - & 103.92 & 0.04 \\
\hline $\mathrm{C} 19 n / \mathrm{C} 19 \mathrm{r}$ & 41.390 & $\begin{array}{l}102.63 \\
(+0.15)\end{array}$ & 109.86 & $\begin{array}{c}101.32 \\
(-0.56)\end{array}$ & 111.27 & $\begin{array}{l}101.27 \\
(+0.28)\end{array}$ & 111.18 & 110.77 & 0.79 \\
\hline C19r/C20n & 42.301 & $\begin{array}{l}121.56 \\
(+0.01)\end{array}$ & 130.80 & $\begin{array}{l}120.58 \\
(-0.14)\end{array}$ & 130.79 & - & - & 130.79 & 0.00 \\
\hline $\mathrm{C} 20 \mathrm{n} / \mathrm{C} 20 \mathrm{r}$ & 43.432 & $\begin{array}{l}151.27 \\
(+0.00)\end{array}$ & 163.19 & 152.69 & 164.53 & - & - & 163.86 & 0.95 \\
\hline $\mathrm{C} 20 \mathrm{r} / \mathrm{C} 21 \mathrm{n}$ & 45.724 & $\begin{array}{l}197.39 \\
(+0.00)\end{array}$ & 218.40 & - & - & - & - & 218.40 & - \\
\hline$C 21 n / C 21 r$ & 47.349 & $\begin{array}{l}218.06 \\
(+0.00)\end{array}$ & 252.27 & - & - & - & - & 252.27 & - \\
\hline
\end{tabular}

Chron boundary ages are referred from Ogg (2012). ${ }^{*}=$ values in parentheses are differences from the shipboard data. Depth scales in CCSF are based on the splice table provided by Boulila et al. (2018). 